\title{
Enumeration of Golomb Rulers and Acyclic Orientations of Mixed Graphs
}

\author{
Matthias Beck \\ Department of Mathematics \\ San Francisco State University \\ San Francisco, CA, U.S.A. \\ mattbeck@sfsu.edu
}

\author{
Tristram Bogart \\ Departamento de Matematicas \\ Universidad de los Andes \\ Bogota, Colombia \\ tc. bogart22@uniandes.edu.co
}

\author{
Tu Pham \\ Department of Mathematics \\ University of California \\ Riverside, CA, U.S.A \\ pham@math.ucr.edu
}

Submitted: Dec 13, 2011; Accepted: Sep 14, 2012; Published: Oct 4, 2012

Mathematics Subject Classifications: 05A15, 05C15, 11P21, 52C35

\begin{abstract}
Dedicated to our friend and mentor Joseph Gubeladze
on the occasion of his $50^{\text {th }}$ birthday
\end{abstract}

\begin{abstract}
A Golomb ruler is a sequence of distinct integers (the markings of the ruler) whose pairwise differences are distinct. Golomb rulers, also known as Sidon sets and $B_{2}$ sets, can be traced back to additive number theory in the 1930s and have attracted recent research activities on existence problems, such as the search for optimal Golomb rulers (those of minimal length given a fixed number of markings). Our goal is to enumerate Golomb rulers in a systematic way: we study

$$
g_{m}(t):=\#\left\{\mathbf{x} \in \mathbb{Z}^{m+1}: 0=x_{0}<x_{1}<\cdots<x_{m}=t \text {, all } x_{j}-x_{k} \text { distinct }\right\},
$$

the number of Golomb rulers with $m+1$ markings and length $t$. Our main result is that $g_{m}(t)$ is a quasipolynomial in $t$ which satisfies a combinatorial reciprocity theorem: $(-1)^{m-1} g_{m}(-t)$ equals the number of rulers $\mathbf{x}$ of length $t$ with $m+1$ markings, each counted with its Golomb multiplicity, which measures how many combinatorially different Golomb rulers are in a small neighborhood of x. Our reciprocity theorem can be interpreted in terms of certain mixed graphs associated to Golomb rulers; in this language, it is reminiscent of Stanley's reciprocity theorem for chromatic polynomials. Thus in the second part of the paper we develop an
\end{abstract}


analogue of Stanley's theorem to mixed graphs, which connects their chromatic polynomials to acyclic orientations.

Keywords: Golomb ruler; Sidon set; combinatorial reciprocity theorem; lattice point; inside-out polytope; Ehrhart quasipolynomial; mixed graph; proper coloring; acyclic orientation

\section{Introduction}

A Golomb ruler is a sequence of $n$ distinct integers whose pairwise differences are distinct: one can picture an actual ruler with $n$ markings having the property that all possible measurements are of distinct length. Golomb rulers have natural applications to phased array radio antennas [2], x-ray analysis of crystal structures [5], and error-correcting codes [13]. Golomb rulers also go by the names of Sidon sets and $B_{2}$ sets; they can be traced back to additive number theory in the 1930s $[9,18]$ (see also the survey article [15]).

In the more recent past, researchers have typically studied existence problems, such as the search for optimal Golomb rulers (those of minimal length given a fixed number of markings), often with an eye toward computational complexity. Figure 1 shows an optimal ruler of length 6 . See, e.g., $[1,6,7,14,17]$ and the parallel-search project on Golomb rulers at http://www.distributed.net/Projects.

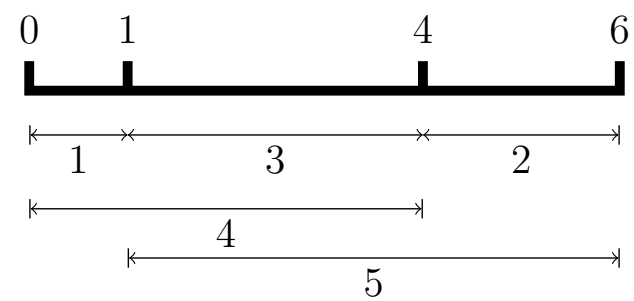

Figure 1: An optimal Golomb ruler with four markings.

Our goal is to enumerate Golomb rulers in a systematic way. We define

$$
g_{m}(t):=\#\left\{\mathbf{x} \in \mathbb{Z}^{m+1}: 0=x_{0}<x_{1}<\cdots<x_{m-1}<x_{m}=t \text {, all } x_{j}-x_{k} \text { distinct }\right\},
$$

the number of Golomb rulers with $m+1$ markings and length $t$. Most often it will be more convenient for us to express this counting function in the equivalent form

$$
g_{m}(t)=\#\left\{\mathbf{z} \in \mathbb{Z}_{>0}^{m}: \begin{array}{l}
z_{1}+z_{2}+\cdots+z_{m}=t, \\
\sum_{j \in U} z_{j} \neq \sum_{j \in V} z_{j} \text { for all dpcs } U, V \subset[m]
\end{array}\right\}
$$

where $[m]:=\{1,2, \ldots, m\}$, and dpcs stands for disjoint proper consecutive subsets, i.e., two sets of the form $\{a, a+1, a+2, \ldots, b\},\{c, c+1, c+2, \ldots, d\}$ for some $1 \leqslant a \leqslant b<$ $c \leqslant d \leqslant m$. 
Motivated by (1), we call a point $\mathbf{z} \in \mathbb{Z}_{\geqslant 0}^{m}$ with $z_{1}+z_{2}+\cdots+z_{m}=t$ a ruler of length $t$; thus a Golomb ruler is a ruler with positive entries satisfying $\sum_{j \in U} z_{j} \neq \sum_{j \in V} z_{j}$ for all dpcs $U, V \subset[m]$.

We define a real Golomb ruler (with $m+1$ markings and length $t$ ) as a vector $\mathbf{z} \in \mathbb{R}_{\geqslant 0}^{m}$ satisfying

$$
z_{1}+z_{2}+\cdots+z_{m}=t \quad \text { and } \quad \sum_{j \in U} z_{j} \neq \sum_{j \in V} z_{j} \text { for all dpcs } U, V \subset[m] .
$$

Two real Golomb rulers $\mathbf{z}, \mathbf{w} \in \mathbb{R}_{\geqslant 0}^{m}$ are combinatorially equivalent if for any dpcs $U, V \subset$ $[m]$,

$$
\sum_{j \in U} z_{j}<\sum_{j \in V} z_{j} \quad \Longleftrightarrow \quad \sum_{j \in U} w_{j}<\sum_{j \in V} w_{j},
$$

in plain English: if their possible measurements satisfy the same order relations. We define the Golomb multiplicity of a ruler $\mathbf{z} \in \mathbb{Z}_{\geqslant 0}^{m}$ to be the number of combinatorially different real Golomb rulers in an $\epsilon$-neighborhood of $\mathbf{z}$ (viewed as a point in $\mathbb{R}^{m}$ ), for sufficiently small $\epsilon>0$. Thus a Golomb ruler is a ruler with Golomb multiplicity 1 .

A quasipolynomial of degree $d$ is a function of the form $c_{d}(t) t^{d}+c_{d-1}(t) t^{d-1}+\cdots+c_{0}(t)$, where the $c_{j}(t)$ are periodic functions in $t$. The lcm of their periods is called the period of this quasipolynomial. Our main result about $g_{m}(t)$ is the following combinatorial reciprocity theorem.

Theorem 1. The Golomb counting function $g_{m}(t)$ is a quasipolynomial in $t$ of degree $m-1$ with leading coefficient $\frac{1}{(m-1) !}$. Its evaluation $(-1)^{m-1} g_{m}(-t)$ equals the number of rulers in $\mathbb{Z}_{\geqslant 0}^{m}$ of length $t$, each counted with its Golomb multiplicity. Furthermore, $(-1)^{m-1} g_{m}(0)$ equals the number of combinatorially different Golomb rulers with $m+1$ markings.

Example 2. Let $m=3$. Using James B. Shearer's Fortran code for Golomb rulers, ${ }^{1}$ we computed the values of $g_{3}(t)$ for $6 \leqslant t \leqslant 35$. Note that if $\mathbf{z}$ is a Golomb ruler with $m+1$ markings, then there is a complementary Golomb ruler $\mathbf{z}^{\prime}$ given by $z_{i}^{\prime}=z_{m-i}$, and $\left(\mathbf{z}^{\prime}\right)^{\prime}=\mathbf{z}$. The rulers $\mathbf{z}$ and $\mathbf{z}^{\prime}$ are always distinct unless $m=1$. Shearer's code computes only one of each complementary pair, but in Table 1 we double the output values to account for both. We also note that by inspection, $g_{3}(t)=0$ for $t<6$.

In Section 2 we show that the period of the quasipolynomial function $g_{3}(t)$ divides 12 and that its leading term is $\frac{1}{2} t^{2}$. Thus from the 24 values $g_{3}(0), g_{3}(1), \ldots, g_{3}(23)$ we can obtain all of the coefficients by interpolation. The remaining 12 values $g_{3}(24), \ldots, g_{3}(35)$ are consistent with the result of this computation, which is:

$$
g_{3}(t)=\left\{\begin{array}{ll}
\frac{1}{2} t^{2}-4 t+10 & \text { if } t \equiv 0 \\
\frac{1}{2} t^{2}-3 t+\frac{5}{2} & \text { if } t \equiv 1,5,7,11 \\
\frac{1}{2} t^{2}-4 t+6 & \text { if } t \equiv 2,10 \\
\frac{1}{2} t^{2}-3 t+\frac{9}{2} & \text { if } t \equiv 3,9 \\
\frac{1}{2} t^{2}-4 t+8 & \text { if } t \equiv 4,6,8
\end{array} \quad(\bmod 12)\right.
$$

${ }^{1}$ Available at http://www.research.ibm.com/people/s/shearer/programs/grs1.txt 


\begin{tabular}{|c|c||c|c||c|c|}
\hline$t$ & $g_{3}(t)$ & $t$ & $g_{3}(t)$ & $t$ & $g_{3}(t)$ \\
\hline 6 & 2 & 16 & 72 & 26 & 240 \\
7 & 6 & 17 & 96 & 27 & 288 \\
8 & 8 & 18 & 98 & 28 & 288 \\
9 & 18 & 19 & 126 & 29 & 336 \\
10 & 16 & 20 & 128 & 30 & 338 \\
11 & 30 & 21 & 162 & 31 & 390 \\
12 & 34 & 22 & 160 & 32 & 392 \\
13 & 48 & 23 & 198 & 33 & 450 \\
14 & 48 & 24 & 202 & 34 & 448 \\
15 & 72 & 25 & 240 & 35 & 510 \\
\hline
\end{tabular}

Table 1: The Golomb counting function $g_{3}(t)$ for $6 \leqslant t \leqslant 35$.

In particular, the coefficient $c_{1}(t)$ has period 2 . The coefficient $c_{0}(t)$ has period 12 but obeys the same formula for $t \equiv j(\bmod 12)$ as for $t \equiv-j(\bmod 12)$ for each $j$. Note also that $g_{3}(0)=10$; Theorem 1 predicts that there are ten combinatorially different Golomb rulers, as we will see in Section 2.

We prove Theorem 1 geometrically, making use of the machinery of inside-out polytopes [4]. This approach leads us to associate a mixed graph (i.e., a graph that may contain both undirected and directed edges; see, e.g., [12]) to the set of all Golomb rulers with a fixed number of markings. In this language, Theorem 1 has an interpretation in terms of acyclic orientations of mixed graphs, which might be of independent interest (Theorem 5 and Corollary 6, proved in Section 3). This reinterpretation is reminiscent of Stanley's reciprocity theorem for the chromatic polynomial of a graph [21]. This leads us to a natural analogue of Stanley's theorem for (general) mixed graphs, as follows.

We write a mixed graph $G$ as $G=(V, E, A)$ where $E$ contains undirected edges and $A$ the directed edges. (We assume that $G$ is simple: that is, that it contains neither multiple edges - directed or undirected-nor loops.) A t-coloring of a mixed graph $G$ is a map $c: V \rightarrow[t]$. Such a $t$-coloring is proper if

- $c(v) \neq c(w)$ for all $\{v, w\} \in E$, and

- $c(v)<c(w)$ for all $(v, w) \in A$.

As with undirected graphs, the chromatic number is the minimum $t$ such that $G$ can be $t$-colored. This parameter was introduced for mixed graphs by Hansen, Kuplinsky, and de Werra [11], who discuss bounds, algorithms, and applications to scheduling problems. Let $\chi_{G}(t)$ denote the number of proper $t$-colorings of $G$. Sotskov and Tanaev [19] (see also [20] for a more recent article) showed that this function (if not identically zero) is a polynomial of degree $|V|$ and computed the two leading coefficients.

An orientation of a mixed graph is obtained by keeping each directed edge and orienting each undirected edge; an orientation is acyclic if it does not contain any coherently- 
oriented cycles. A coloring $c$ and an orientation of $G$ are compatible if

$$
c(u) \leqslant c(v) \quad \Longleftrightarrow \quad u \rightarrow v .
$$

Note that $c$ may not be a proper coloring.

Here is our generalization of Stanley's chromatic reciprocity theorem to mixed graphs. Our approach (in Section 4) also yields a new proof of polynomiality of $\chi_{G}(t)$.

Theorem 3. Let $G$ be a mixed graph. For a positive integer $t,(-1)^{|V|} \chi_{G}(-t)$ equals the number of t-colorings of $G$, each counted with multiplicity equal to the number of compatible acyclic orientations of $G$.

Corollary 4. For $G$ a mixed graph, $(-1)^{|V|} \chi_{G}(-1)$ equals the number of acyclic orientations of $G$.

In Section 5 we end with some open questions about Golomb rulers suggested by our geometric approach.

\section{Inside-out Polytopes}

It is a short step to interpret a Golomb ruler in its measurement representation (as in (1)) as an integer lattice point confined to the positive orthant in $\mathbb{R}^{m}$ and the affine space defined through

$$
z_{1}+z_{2}+\cdots+z_{m}=t
$$

More precisely, a Golomb ruler with $m+1$ markings and length $t$ is a lattice point in the $t$ 'th dilate of

$$
\Delta_{m}^{\circ}:=\left\{\mathbf{z} \in \mathbb{R}_{>0}^{m}: z_{1}+z_{2}+\cdots+z_{m}=1\right\},
$$

the $(m-1)$-dimensional open standard simplex living in $\mathbb{R}^{m}$. However, this description does not yet capture the distinctness condition: a Golomb-ruler lattice point must also avoid the hyperplanes in $\mathbb{R}^{m}$ given by equations of the form

$$
\sum_{j \in U} z_{j}=\sum_{j \in V} z_{j}
$$

for all dpcs $U, V \subset[m]$. Let $\mathcal{G}_{m}$ be the collection of all such hyperplanes. Thus we have built a geometric setting in which to compute the Golomb counting function as

$$
g_{m}(t)=\#\left(\left(t \Delta_{m}^{\circ} \backslash \mathcal{G}_{m}\right) \cap \mathbb{Z}^{m}\right) .
$$

In the language of [4], $\left(\Delta_{m}, \mathcal{G}_{m}\right)$ is an inside-out polytope ${ }^{2}$ and $g_{m}(t)$ is the (open) Ehrhart quasipolynomial of $\left(\Delta_{m}, \mathcal{G}_{m}\right)$. Figure 2 shows this geometric setting (viewed in the plane $\left.z_{1}+z_{2}+z_{3}=1\right)$ for the case $m=3$.

\footnotetext{
${ }^{2}$ To be $100 \%$ precise, [4] would compute $g_{m}(t)$ as $\#\left(\left(\Delta_{m}^{\circ} \backslash \mathcal{G}_{m}\right) \cap \frac{1}{t} \mathbb{Z}^{m}\right)$, but this is equivalent as the hyperplanes in $\mathcal{G}_{m}$ are linear.
} 


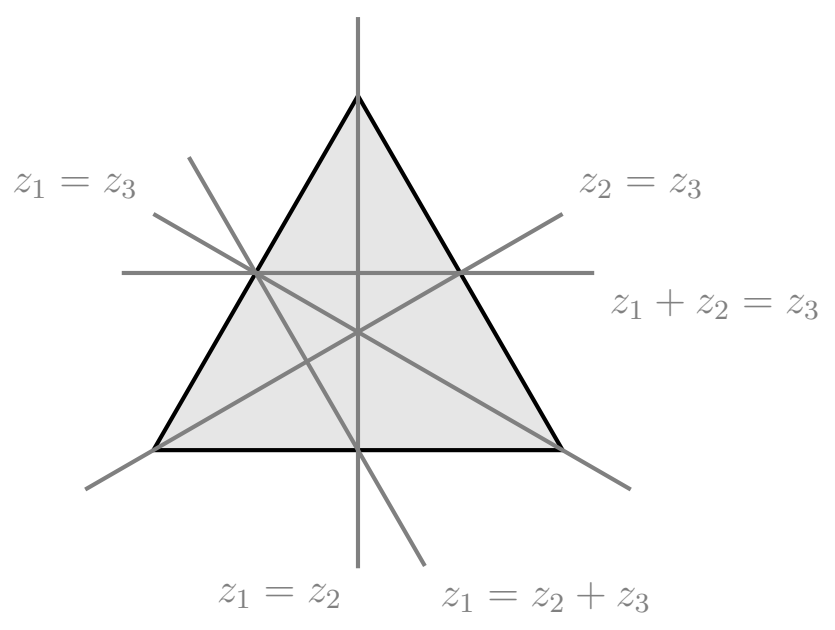

Figure 2: The inside-out polytope $\left(\Delta_{3}, \mathcal{G}_{3}\right)$.

The vertices of this inside-out polytope are

$$
(0,0,1),\left(\frac{1}{2}, 0, \frac{1}{2}\right),\left(\frac{1}{4}, \frac{1}{4}, \frac{1}{2}\right),\left(0, \frac{1}{2}, \frac{1}{2}\right),\left(\frac{1}{3}, \frac{1}{3}, \frac{1}{3}\right),\left(\frac{1}{2}, \frac{1}{4}, \frac{1}{4}\right),(1,0,0),\left(\frac{1}{2}, \frac{1}{2}, 0\right),(0,1,0) .
$$

The lcm of the denominators among all of these coordinates is twelve. This shows that the period of the quasipolynomial $g_{3}(t)$ divides twelve, as claimed in Example 2. We can also see that $\mathcal{G}_{3}$ dissects $\Delta_{3}$ into ten polygons, which correspond to the combinatorially different Golomb rulers.

The general setup of an inside-out polytope $(\mathcal{P}, \mathcal{H})$ consists of a rational polytope $\mathcal{P}$ and a rational hyperplane arrangement $\mathcal{H}$ in $\mathbb{R}^{d}$; that is, the linear equations and inequalities defining $\mathcal{P}$ and $\mathcal{H}$ have integer coefficients. The goal is to compute the counting function

$$
L_{\mathcal{P}, \mathcal{H}}^{\circ}(t):=\#\left((\mathcal{P} \backslash \mathcal{H}) \cap \frac{1}{t} \mathbb{Z}^{d}\right),
$$

and it follows from Ehrhart's theory of counting lattice points in dilates of rational polytopes $[3,8]$ that this function is a quasipolynomial in $t$ whose degree is $\operatorname{dim}(P)$, whose (constant) leading coefficient is the normalized lattice volume of $P$, and whose period divides the lcm of all denominators that appear in the coordinates of the vertices of $(\mathcal{P}, \mathcal{H})$. Furthermore, [4] established the reciprocity theorem

$$
L_{\mathcal{P}^{\circ}, \mathcal{H}}^{\circ}(-t)=(-1)^{\operatorname{dim} \mathcal{P}} L_{\overline{\mathcal{P}}, \mathcal{H}}(t)
$$

where $\mathcal{P}^{\circ}$ and $\overline{\mathcal{P}}$ denote the interior and closure of $\mathcal{P}$, respectively, and

$$
L_{\mathcal{P}, \mathcal{H}}(t):=\sum_{\mathbf{m} \in \frac{1}{t} \mathbb{Z}^{d}} \operatorname{mult}_{\mathcal{P}, \mathcal{H}}(\mathbf{m})
$$

where $\operatorname{mult}_{\mathcal{P}, \mathcal{H}}(\mathbf{m})$ denotes the number of closed regions of $(\mathcal{P}, \mathcal{H})$ containing $\mathbf{m}$. (A region of $(\mathcal{P}, \mathcal{H})$ is a connected component of $\mathcal{P} \backslash \mathcal{H}$; a closed region is the closure of a 
region.) It follows from Ehrhart's work and (4) that

$$
L_{\mathcal{P}, \mathcal{H}}(0)=\# \text { regions of }(\mathcal{P}, \mathcal{H}) .
$$

See [4] for this and several more properties of inside-out polytopes. The concept of insideout polytopes has been applied to a number of combinatorial settings; at the heart of any such application is an interpretation of the regions of $(\mathcal{P}, \mathcal{H})$, which we will now give for the Golomb inside-out polytope $\left(\Delta_{m}, \mathcal{G}_{m}\right)$.

Proof of Theorem 1. The first statement follows immediately from the fact that $\left(\Delta_{m}, \mathcal{G}_{m}\right)$ is a rational inside-out polytope and $\Delta_{m}$ is a unimodular $(m-1)$-dimensional simplex.

Viewing (3) from (2), we know that $(-1)^{m-1} g_{m}(-t)$ equals the number of rulers in $\Delta \cap \frac{1}{t} \mathbb{Z}^{m}$, each counted with multiplicity given by the number of closed regions of $\left(\Delta_{m}, \mathcal{G}_{m}\right)$ it lies in. These regions, in turn, are defined by inequalities of the form

$$
\sum_{j \in U} z_{j}<\sum_{j \in V} z_{j}
$$

for some dpcs $U, V \subset[m]$, and thus the multiplicity of a ruler $\mathbf{z}$ is precisely given by the number of combinatorially different real Golomb rulers in a neighborhood of $\mathbf{z}$.

The last statement of Theorem 1 follows from (5).

\section{The Regions of $\left(\Delta_{m}, \mathcal{G}_{m}\right)$}

For each positive integer $m$, we define the Golomb graph $\Gamma_{m}$ to be a mixed graph whose vertices are all proper consecutive subsets of $[m]$. The underlying graph is complete and an edge $U V$ is directed $(U \rightarrow V)$ if and only if $U \subset V$. All other edges are undirected. Acyclic orientations of a Golomb graph allow us to give an interpretation of the regions of $\left(\Delta_{m}, \mathcal{G}_{m}\right)$ in the following sense.

Theorem 5. The regions of the Golomb inside-out polytope $\left(\Delta_{m}, \mathcal{G}_{m}\right)$ are in one-to-one correspondence with the acyclic orientations of the Golomb graph $\Gamma_{m}$ that satisfy the relation

$$
A \rightarrow B \quad \Longleftrightarrow \quad U \rightarrow V .
$$

for all proper consecutive subsets $A$ and $B$ of $[m]$ of the form $A=U \cup W$ and $B=V \cup W$ for some nonempty disjoint sets $U, V, W$

Proof. Let $R$ be a region of $\left(\Delta_{m}, \mathcal{G}_{m}\right)$. Thus $R$ is the intersection of $\Delta_{m}$ with halfspaces defined by inequalities of the form

$$
\sum_{j \in U} z_{j}<\sum_{j \in V} z_{j}
$$

for some dpcs $U, V \subset[m]$. So $U V$ is an undirected edge of $\Gamma_{m}$; assign it the orientation $U \rightarrow V$. 

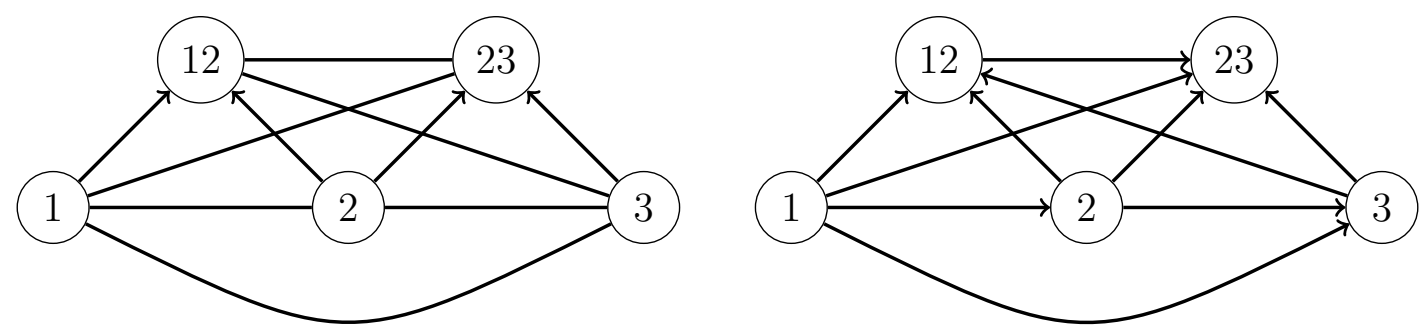

Figure 3: The mixed graph $\Gamma_{3}$ (left) and an acyclic orientation of $\Gamma_{3}$ (right).

The directed edges of $\Gamma_{m}$ are of the form $U V$ for $U \subset V$, so we still need to orient edges of the form $A B$ where $A=U \cup W$ and $B=V \cup W$ for some nonempty disjoint sets $U, V, W$. We orient these according to (6) (note that the corresponding halfspace defined through $\sum_{j \in A} z_{j}<\sum_{j \in B} z_{j}$ is identical to that given by (7)). The resulting orientation of $\Gamma_{m}$ is acyclic since a coherently-oriented cycle gives rise to the nonsensical inequality $\sum_{j \in M} z_{j}<\sum_{j \in M} z_{j}$ for some multiset $M$.

Conversely, suppose we are given an acyclic orientation of $\Gamma_{m}$ that satisfies (6). Index the variables $y_{U}$ of a vector in $\mathbb{R}^{m(m+1) / 2-1}$ by the proper consecutive subsets $U$ of $[m]$ and consider the braid arrangement given by the hyperplanes $y_{U}=y_{V}$ in $\mathbb{R}^{m(m+1) / 2-1}$. It is a famous observation of Greene [10] that the acyclic orientations of a graph are in one-to-one correspondence with the region of the corresponding graphical arrangement (in our case, the above braid arrangement, since our underlying graph is complete). Now consider the function $\mathbb{R}^{m(m+1) / 2-1} \rightarrow \mathbb{R}^{m}$ defined through

$$
y_{U} \mapsto \sum_{j \in U} z_{j}
$$

This is a linear map of full rank, i.e., the regions of the braid arrangement, being fulldimensional sets in the domain, are mapped to full-dimensional sets in the range. Furthermore, since the all-ones vector is in each closed region, we can project further to the affine space defined by $z_{1}+z_{2}+\cdots+z_{m}=1$.

This theorem allows us to rephrase Theorem 1 in terms of acyclic orientations of $\Gamma_{m}$. We call a ruler $\mathbf{z} \in \mathbb{Z}_{\geqslant 0}^{m}$ and an orientation of $\Gamma_{m}$ compatible if

$$
\sum_{j \in U} z_{j} \leqslant \sum_{j \in V} z_{j} \quad \Longleftrightarrow \quad U \rightarrow V
$$

Corollary 6. The evaluation $(-1)^{m-1} g_{m}(-t)$ equals the number of rulers in $\mathbb{Z}_{\geqslant 0}^{m}$ of length $t$, each counted with multiplicity equal to the number of compatible acyclic orientations of $\Gamma_{m}$ that satisfy (6). Furthermore, $(-1)^{m-1} g_{m}(0)$, the number of combinatorially different Golomb rulers, equals the number of acyclic orientations of $\Gamma_{m}$ that satisfy (6). 


\section{Mixed Graph Coloring Reciprocity}

Our proof of Theorem 3 closely follows the proof of Stanley's chromatic reciprocity theorem given in [4]. Given a mixed graph $G=(V, E, A)$ with $V=[n]$, define the polytope

$$
\mathcal{P}(G):=\left\{\mathbf{x} \in[0,1]^{n}: x_{j} \leqslant x_{k} \text { whenever }(j, k) \in A\right\}
$$

and the hyperplane arrangement $\mathcal{H}(G)$ consisting of the hyperplanes $x_{j}=x_{k}$ for all $\left\{v_{j}, v_{k}\right\} \in E$. The following result is the mixed-graph analogue of [10].

Proposition 7. The regions of $\mathbb{R}^{n} \backslash \mathcal{H}(G)$ that intersect $\mathcal{P}(G)$ are in bijection with the acyclic orientations of $G$.

Proof. Let $R$ be an open region of $\mathcal{H}(G)$ that intersects $\mathcal{P}(G)$, hence also intersects $\mathcal{P}(G)^{\circ}$. Then for each hyperplane $x_{i}=x_{j}$ in $\mathcal{H}(G), R$ is entirely on one side, hence determines an orientation $\alpha$ as follows: for each edge $\{i, j\} \in E$, choose $i \rightarrow j$ if $x_{i}<x_{j}$ in $R$, or $j \rightarrow i$ if $x_{i}>x_{j}$ in $R$. In the set $R \cap \mathcal{P}(G)^{\circ}$, we also have $x_{i}<x_{j}$ for all $(i, j) \in A$. Since $R$, being open, intersects the interior of $\mathcal{P}(G)^{\circ}$, all of these inequalities must be consistent. That is, $\alpha$ is acylic.

Since two different regions must be on opposite sides of at least one hyperplane, they determine different orientations. Finally, if $\alpha$ is an ayclic orientation of $G$, choose any total order $\pi$ of the vertices that is consistent with $\alpha$, including the fixed directions on the arrows $A$. Then if $R$ is the region containing the points of $\mathbb{R}^{n}$ that satisfy $x_{\pi_{1}}<\cdots<x_{\pi_{n}}$, then $R$ maps to $\alpha$ under the rule given above. That is, the map from regions to orientations is both injective and surjective.

We say that $G$ is acylic if the directed graph $(G, A)$ contains no directed cycles. Note that in this case there exists at least one total order on the vertices compatible with the directed edges. Orienting the undirected edges according to such an order, we can always obtain an acyclic orientation on all of $G$. Note that if $G$ is not acyclic, then there exist no proper $t$-colorings for any $t$. On the other hand, if $G$ is acyclic then there exist proper $t$-colorings for all sufficiently large $t$, and in particular for all $t \geqslant n$.

Lemma 8. If the mixed graph $G$ is acyclic then the polytope $\mathcal{P}(G)$ is full dimensional.

Proof. The polytope $\mathcal{P}(G)$ depends only on the directed graph $D=(V, A)$. Since $D$ is acyclic, there is a total order $i_{1} \prec i_{2} \prec \cdots \prec i_{n}$ on $[n]$ such that $j \prec k$ for all $\left(v_{j}, v_{k}\right) \in A$. Thus

$$
\mathcal{P}(G) \supseteq\left\{\mathbf{x} \in \mathbb{R}^{n}: 0 \leqslant x_{i_{1}} \leqslant x_{i_{2}} \leqslant \ldots \leqslant x_{i_{n}} \leqslant 1\right\}
$$

which is a full-dimensional simplex in $\mathbb{R}^{n}$, so $\mathcal{P}(G)$ is full-dimensional.

Lemma 9. For any acyclic mixed graph $G$, the inside-out polytope $(\mathcal{P}(G), \mathcal{H}(G))$ is integral.

Proof. Any closed region of $(\mathcal{P}(G), \mathcal{H}(G))$ is defined by a collection of inequalities of the form $x_{j}-x_{k} \leqslant 0$ and $x_{j} \geqslant 0$. In particular, the left-hand sides of these inequalities are all vectors of the form $\mathbf{e}_{j}-\mathbf{e}_{k}$ or $-\mathbf{e}_{j}$. Any matrix whose rows are all such vectors is totally unimodular. This implies that all vertices of the region are integral [16]. 
Proof of Theorem 3. Set $\mathcal{P}:=\mathcal{P}(G)$ and $\mathcal{H}:=\mathcal{H}(G)$. Using the identification of maps $c: V \rightarrow \mathbb{Z}$ with lattice points in $\mathbb{R}^{n}$, we see that for $t \geqslant 1, c$ is a $(t-1)$-coloring (respecting the directed edges $A$ ) if $c \in t \mathcal{P}^{\circ}(G)$ and $c \notin \mathcal{H}$. That is, $\chi_{G}(t-1)=E_{\mathcal{P}^{\circ}, \mathcal{H}}^{\circ}(t)$, which equals $(-1)^{n} E_{\mathcal{P}, \mathcal{H}}(-t)$ by $(3)$. Using Lemma 9 , we see that the functions $E_{\mathcal{P}, \mathcal{H}}$ and $E_{\mathcal{P}}^{\circ}, \mathcal{H}$ are polynomials and hence the reciprocity extends to all values of $t$. In particular, for

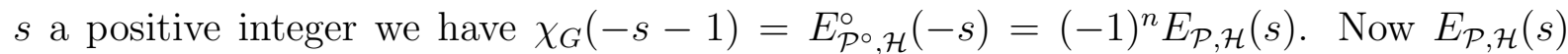
counts each point $c \in s \mathcal{P}$ with multiplicity equal to the number of closed regions of $\mathcal{H}$ that intersect the interior of $\mathcal{P}$ and contain $c$. By Proposition 7 , regions correspond to acyclic orientations of $G$. The closed region corresponding to an orientation $\alpha$ contains $c$ if and only if $c(u) \leqslant c(v)$ for each edge $u \rightarrow v$ in $\alpha$.

Example 10. Let $G=([3],\{\{1,3\},\{2,3\}\},\{(1,2)\})$, a triangle with one directed edge and two undirected edges. Of the four orientations of $G$, three are acyclic and each of these three determines a total order $\prec$ on the vertices. These three orders are:

$$
1 \prec 2 \prec 3,1 \prec 3 \prec 2,3 \prec 1 \prec 2 \text {. }
$$

The polytope $\mathcal{P}(G)$ is the half-cube given by the five inequalities

$$
0 \leqslant x_{1} \leqslant x_{2} \leqslant 1,0 \leqslant x_{3} \leqslant 1 \text {. }
$$

The arrangement $\mathcal{H}(G)$ consists of the two hyperplanes $x_{1}=x_{3}$ and $x_{2}=x_{3}$, and the inside-out polytope $(\mathcal{P}(G), \mathcal{H}(G))$ has three open regions in bijection with the acyclic orientations of $G$ :

$$
\begin{aligned}
& R_{123}=\left\{\mathbf{x} \in \mathbb{R}^{3}: 0<x_{1}<x_{2}<x_{3}<1\right\} \\
& R_{132}=\left\{\mathbf{x} \in \mathbb{R}^{3}: 0<x_{1}<x_{3}<x_{2}<1\right\} \\
& R_{312}=\left\{\mathbf{x} \in \mathbb{R}^{3}: 0<x_{3}<x_{1}<x_{2}<1\right\}
\end{aligned}
$$

For this graph it is easy to compute $\chi_{G}(t)$ directly. In any coloring $c$, we must choose $c(1)<c(2)$ and then we can choose $c(3)$ to be any remaining color. Thus there are $\left(\begin{array}{l}t \\ 2\end{array}\right)(t-2)=\frac{t(t-1)(t-2)}{2} t$-colorings for any nonnegative integer $t$. Then $(-1)^{3} \chi_{G}(-1)=$ $-\frac{(-1)(-2)(-3)}{2}=3$, which is the number of regions of $(\mathcal{P}(G), \mathcal{H}(G))$ as stated in Theorem 3 .

As we know from the proof of Theorem 3, we can also use Ehrhart reciprocity to interpret the evaluation of $\chi_{G}$ at other negative integers. Specifically, $-\chi_{G}(-t)$ is the number of lattice points in $(t-1) \mathcal{P}$ with multiplicities, where the multiplicity of each point is the number of closed regions of the inside-out polytope that contain it. For instance, $-\chi_{G}(-2)=(-1) \frac{(-2)(-3)(-4)}{2}=12$ and $-\chi_{G}(-3)=(-1) \frac{(-3)(-4)(-4)}{2}=30$. In Table 2, we list the lattice points in $\mathcal{P}$ and $2 \mathcal{P}$ and the regions that contain each point. Indeed, the multiplicities add up to 12 and 30, respectively.

Note that in this example, $\mathcal{H}(G)$ is not transverse to $\mathcal{P}(G)$. The two hyperplanes $x_{1}=x_{3}, x_{2}=x_{3}$ in $\mathcal{H}(G)$ meet in a flat given by $x_{1}=x_{2}=x_{3}$. This flat intersects only the boundary of $\mathcal{P}$ since the equation $x_{1}=x_{2}$ is a supporting hyperplane of $\mathcal{P}$. 


\begin{tabular}{|c|c|c|}
\hline point $\mathbf{z} \in \mathcal{P} \cap \mathbb{Z}^{3}$ & regions containing $\mathbf{z}$ & multiplicity of $\mathbf{z}$ \\
\hline$(0,0,0)$ & $R_{123}, R_{132}, R_{312}$ & 3 \\
$(0,0,1)$ & $R_{123}$ & 1 \\
$(0,1,0)$ & $R_{132}, R_{312}$ & 2 \\
$(0,1,1)$ & $R_{123}, R_{132}$ & 2 \\
$(1,1,0)$ & $R_{312}$ & 1 \\
$(1,1,1)$ & $R_{123}, R_{132}, R_{312}$ & 3 \\
\hline
\end{tabular}

\begin{tabular}{|c|c|c|}
\hline point $\mathbf{z} \in 2 \mathcal{P} \cap \mathbb{Z}^{3}$ & regions containing $\mathbf{z}$ & multiplicity of $\mathbf{z}$ \\
\hline$(0,0,0)$ & $R_{123}, R_{132}, R_{312}$ & 3 \\
$(0,0,1)$ & $R_{123}$ & 1 \\
$(0,0,2)$ & $R_{123}$ & 1 \\
$(0,1,0)$ & $R_{132}, R_{312}$ & 2 \\
$(0,1,1)$ & $R_{123}, R_{132}$ & 2 \\
$(0,1,2)$ & $R_{123}$ & 1 \\
$(0,2,0)$ & $R_{132}, R_{312}$ & 2 \\
$(0,2,1)$ & $R_{132}$ & 1 \\
$(0,2,2)$ & $R_{132}, R_{132}$ & 2 \\
$(1,1,0)$ & $R_{312}$ & 1 \\
$(1,1,1)$ & $R_{123}, R_{132}, R_{312}$ & 3 \\
$(1,1,2)$ & $R_{123}$ & 1 \\
$(1,2,0)$ & $R_{312}$ & 1 \\
$(1,2,1)$ & $R_{132}, R_{312}$ & 2 \\
$(1,2,2)$ & $R_{123}, R_{132}$ & 2 \\
$(2,2,0)$ & $R_{312}$ & 1 \\
$(2,2,1)$ & $R_{312}$ & 1 \\
$(2,2,2)$ & $R_{123}, R_{132}, R_{312}$ & 3 \\
\hline
\end{tabular}

Table 2: Lattice points in $\mathcal{P}$ and $2 \mathcal{P}$ and the regions that contain each point.

\section{Open Problems}

The search for optimal Golomb rulers remains a challenging open problem. This problem is related to our paper: the smallest positive integer that is not a root of $g_{m}(t)$ gives the length of an optimal Golomb ruler with $m+1$ markings. Our methods present new open problems, from the computation of $g_{m}(t)$ to specific questions, e.g., about the period of $g_{m}(t)$ and the number of combinatorially different Golomb rulers with a given number of markings (this sequence starts with 1, 2, 10, 114, 2608, and 107498). Our reciprocity theorem for chromatic polynomials of mixed graphs suggest open problems about these polynomials, from classification problems (which polynomials are chromatic polynomials of mixed graphs?) to interpretations of and relations between its coefficients. 


\section{Acknowledgements}

We thank Thomas Zaslavsky for helpful comments on an earlier version of this manuscript.

\section{References}

[1] Roger C. Alperin and Vladimir Drobot. Golomb rulers. Math. Mag., 84:48-55, 2011.

[2] Wallace C. Babcock. Intermodulation interference in radio systems/frequency of occurrence and control by channel selection. Bell System Technical J., 31:63-73, 1953.

[3] Matthias Beck and Sinai Robins. Computing the continuous discretely: Integer-point enumeration in polyhedra. Undergraduate Texts in Mathematics. Springer, New York, 2007. Electronically available at http://math.sfsu.edu/beck/ccd.html.

[4] Matthias Beck and Thomas Zaslavsky. Inside-out polytopes. Adv. Math., 205(1):134162, 2006. arXiv:math. C0/0309330.

[5] Gary S. Bloom and Solomon W. Golomb. Application of numbered unidirected graphs. Proceedings of the IEEE, 65:562-570, 1977.

[6] Apostolos Dollas, William T. Rankin, and David McCracken. A new algorithm for Golomb ruler derivation and proof of the 19 mark ruler. IEEE Trans. Inform. Theory, 44(1):379-382, 1998.

[7] Konstantinos Drakakis. A review of the available construction methods for Golomb rulers. Adv. Math. Commun., 3(3):235-250, 2009.

[8] Eugène Ehrhart. Sur les polyèdres rationnels homothétiques à $n$ dimensions. $C . R$. Acad. Sci. Paris, 254:616-618, 1962.

[9] Paul Erdős and Pál Turán. On a problem of Sidon in additive number theory, and on some related problems. J. London Math. Soc., 16:212-215, 1941.

[10] Curtis Greene. Acyclic orientations. In M. Aigner, editor, Higher Combinatorics, volume 31 of NATO Adv. Study Inst. Ser., Ser. C: Math. Phys. Sci., pages 65-68. Reidel, Dordrecht, 1977.

[11] Pierre Hansen, Julio Kuplinsky, and Dominique de Werra. Mixed graph colorings. Math. Methods Oper. Res., 45(1):145-160, 1997.

[12] Frank Harary and Ed Palmer. Enumeration of mixed graphs. Proc. Amer. Math. Soc., 17:682-687, 1966.

[13] Torleiv Kløve. Bounds and construction for difference triangle sets. IEEE Trans. Inform. Theory, 35(4):879-886, 1989.

[14] Christophe Meyer and Periklis A. Papakonstantinou. On the complexity of constructing Golomb rulers. Discrete Appl. Math., 157(4):738-748, 2009.

[15] Kevin O'Bryant. A complete annotated bibliography of work related to Sidon sequences. Electron. J. Combin. Dynamic Survey 11, 2004. 
[16] Alexander Schrijver. Combinatorial Optimization. Polyhedra and Efficiency. Vol. $A-C$, volume 24 of Algorithms and Combinatorics. Springer-Verlag, Berlin, 2003.

[17] James B. Shearer. Some new disjoint Golomb rulers. IEEE Trans. Inform. Theory, 44(7):3151-3153, 1998.

[18] Simon Sidon. Ein Satz über trigonometrische Polynome und seine Anwendung in der Theorie der Fourier-Reihen. Math. Ann., 106(1):536-539, 1932.

[19] Ju. N. Sotskov and V. S. Tanaev. Chromatic polynomial of a mixed graph. Vescī Akad. Navuk BSSR Ser. Fīz.-Mat. Navuk, (6):20-23, 140, 1976.

[20] Yuri N. Sotskov, Vjacheslav S. Tanaev, and Frank Werner. Scheduling problems and mixed graph colorings. Optimization, 51(3):597-624, 2002.

[21] Richard P. Stanley. Acyclic orientations of graphs. Discrete Math., 5:171-178, 1973. 